\title{
Double Contrast-enhanced Ultrasound for the Preoperative Gross Classification of Gastric Cancer: a Comparison With Multidetector Computed Tomography
}

\section{Ping He}

Peking University Third Hospital

\section{Lan Zeng}

Peking University Third Hospital

\section{Liying Miao}

Peking University Third Hospital

\section{Tianli Wang}

Peking University Third Hospital

Juxiang Ye

Peking University Third Hospital

\section{Lingmei Meng}

Peking University Third Hospital

\section{Heng Xue}

Peking University Third Hospital

\section{Fan Zhang}

Peking University Third Hospital

\section{Bo Zhao}

Peking University Third Hospital

Huiyu Ge ( $\sigma$ ghyzmw@163.com )

Peking University Third Hospital

\section{Research Article}

Keywords: Gastric cancer, Double contrast-enhanced ultrasound, Multidetector computed tomography, Gross classification

Posted Date: October 20th, 2021

DOI: https://doi.org/10.21203/rs.3.rs-983579/v1 
License: (c) (i) This work is licensed under a Creative Commons Attribution 4.0 International License. Read Full License 


\section{Abstract}

Purpose To compare the diagnostic performance of double contrast-enhanced ultrasound (DCEUS) and multi-detector row computed tomography (MDCT) in the gross classification of gastric cancer (GC) preoperatively.

Methods 54 patients with GC proved by histology were included in this study. The sensitivity and specificity of DCEUS and MDCT for gross classification were calculated and compared. The area under the curve (AUC) from a receiver operating characteristic curve analysis was used to evaluate the difference of the diagnostic performance between these two methods.

Results There were no significant differences between DCEUS and MDCT in terms of AUC values for early

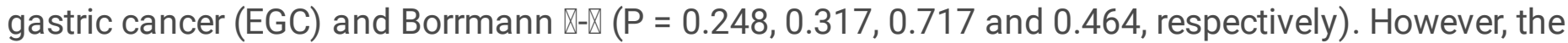
sensitivities of DCEUS for EGC, Borrmann $\otimes$ and Borrmann $\otimes$ were higher than those of MDCT (75\% versus $62 \% ; 100 \%$ versus $50 \% ; 90 \%$ versus $73 \%$ ). The specificity of DCEUS for Borrmann $\otimes$ was lower than that of MDCT ( $50 \%$ versus $75 \%$ ). The AUC value of MDCT for Borrmann $₫$ was significantly higher than that of DCEUS $(0.927$ versus $0.625 ; \mathrm{P}=0.001)$. The accuracy and specificity of DCEUS and MDCT for Borrmann $\rrbracket$ were similar, but the sensitivity of MDCT was significantly higher than that of DCEUS ( $88 \%$ versus $25 \%$ ).

Conclusion DCEUS may be considered as a useful complementary imaging modality to MDCT for the evaluation of the gross classification of GC preoperatively.

\section{Introduction}

Gastric cancer (GC) is one of the most common cancers worldwide, the prognosis of which is closely related to the gross appearance [1-3]. According to the Japanese Gastric Cancer Association criteria, the gross appearances of gastric cancers were classified into two types: early gastric cancer (EGC) and advanced gastric cancer (AGC) [4]. Macroscopic Borrmann type for AGC, developed in 1926, is still a valuable clinicopathological characteristic and used by pathologists and surgeons worldwide, because it can easily be decided by macroscopic pathological examination after excision $[2,5]$. The precise preoperative diagnosis and gross classification is important to the optimal treatment of GC.

Many modalities, such as multidetector computed tomography (MDCT), endoscopic ultrasound, and magnetic resonance imaging, have been used for assessing the gross classification of GC. Double contrast-enhanced ultrasound (DCEUS), in which intravenous contrast enhanced ultrasound is combined with oral contrast-enhanced ultrasound, is an accurate, well-tolerated, noninvasive diagnostic method for preoperative evaluation of GC [2-3,6-8]. In addition to advantages, such as convenience, low cost, and no radiation, oral contrast-enhanced ultrasound can clearly display the stratification of gastric wall by filling the stomach with oral contrast agents, such as water. Intravenous contrast enhanced ultrasound can be used to evaluate the microvessels and tissue perfusion, which proves to be successful in solid organs such as the liver and kidney [9-10]. It has many advantages, such as no toxicity to liver or kidney, low risk of allergy, and no radiation. 
Studies about the comparison of DCEUS and MDCT in the gross classification of GCs are limited [2]. The purpose of this study was to compare the diagnostic performance of DCEUS and MDCT in the gross classification of GCs preoperatively.

\section{Material And Methods}

\section{Patients}

From December 2011 to January 2015, a total of 54 patients (36 men and 18 women, mean age $61 \pm 9.70$ y) with GC proven by endoscopic biopsy were examined using CEUS and MDCT preoperatively. Surgical excision was performed within a week after both examinations. This patient cohort was already published in another study, which was about the tumor staging of GC (blinded reference).

\section{DCEUS}

The ultrasound examinations were performed using Philips iU22 system (Philips Healthcare, Bothell, WA) equipped with convex-array transducers (C5-1) and linear transducer (L9-3). The exams were carried out after fasting for at least 6 hours. Patients were asked to drink about $500-800 \mathrm{~mL}$ of water as quickly as possible, which dilates the stomach and displaces the air within it. If possible, L9-3 transducer was used to get a better resolution. Intravenous CEUS was performed with convex transducers with low mechanical index of 0.06-0.08. A 2.4-mL bolus of SonoVue (Bracco SpA, Milan, Italy) was injected through superficial elbow vein, followed by a $5-\mathrm{mL}$ saline flush. The intravenous contrast study could be repeated a second time with a time interval of at least 15 minutes, if necessary. All DCEUS were performed and interpreted by one of the two radiologists (H.Y.G. and L.Y.M., with 17 years and 29 years of experience in gastroenteric imaging respectively, and 14 years of experience in CEUS imaging). Both of them were blinded to MDCT results but aware of the presence of GC. A consensus was reached by discussion in cases of disagreement.

\section{MDCT}

MDCT (Somatom Definition Flash, Siemens Medical Solutions, Forchheim, Germany) was used for CT scanning. Patients needed to fast for at least 6 hours. In order to dilate the stomach, patients should drink approximately $600-1000 \mathrm{~mL}$ of water $5 \mathrm{~min}$ before CT examination. An intravenous dose of $80 \mathrm{~mL}$ of contrast material (ioversol, $350 \mathrm{mg} / \mathrm{mL}$, Mallinckrodt Canada ULC, Quebec, Canada) was injected at a rate of $3 \mathrm{~mL} / \mathrm{s}$. All images of MDCT were reviewed by the same radiologist (TLW, with 30 years of experience in gastroenteric imaging), who was blinded to the CEUS findings but aware of the presence of $\mathrm{GC}$.

\section{Pathologic analysis}

All resected specimens were examined by one of the two experienced pathologists (LMM and JXY, with 11 years and 8 years of experience in the field of gastroenteric tumor pathologic diagnosis) who were 
unaware of the DCEUS and MDCT findings. A consensus was reached by discussion in cases of disagreement.

EGC was defined as a tumor limited to the mucosa or submucosa, independent of lymph node status, whereas AGC was defined as a tumor invading the muscularis propria or deeper. AGC was further classified into four growth types according to the Borrmann criteria: type I, polypoid tumor; type II, ulcerative lesion with elevated and sharply demarcated margins; type III, ulcerative lesion without definite limits, infiltrating into the surrounding gastric wall; type IV, diffusely infiltrating tumor without ulcer or a discretely marginated mass [11].

\section{Statistical analysis}

The statistical analysis was performed with MedCalc version 14.8.1.0 software (MedCalc Software, Mariakerke, Belgium). Continuous variables were expressed as means \pm standard deviations. The accuracy, sensitivity, specificity, and Youden's index were calculated with DCEUS and MDCT for gross classification. The area under the curve (AUC) from a receiver operating characteristic curve analysis was used to evaluate the difference of the diagnostic performance between these two methods. For all analyses, $p$ values less than 0.05 was considered statistically significant.

\section{Results}

Table 1 shows the accuracy, sensitivity, specificity, and Youden index of DCEUS and MDCT in determining gross classification of GC. Table 2 reveals the AUC values for each gross classification of GC. There were no significant differences between DCEUS and MDCT in terms of AUC values for EGC and Borrmann $\mathbb{X}-\mathbb{X}$ $(P=0.248,0.317,0.717$ and 0.464 , respectively). However, the sensitivities of DCEUS for EGC, Borrmann $\triangle$ and Borrmann $₫$ were higher than those of MDCT (75\% versus $62 \% ; 100 \%$ versus $50 \% ; 90 \%$ versus $73 \%$; Figs. 1, 2 and 4). The specificity of DCEUS for Borrmann $₫$ was lower than that of MDCT (50\% versus $75 \%$ ). The sensitivities of DCEUS and MDCT for Borrmann $₫$ were both very low (17\% versus $33 \%$; Fig. 3 ). The AUC value of MDCT for Borrmann $\otimes$ was significantly higher than that of DCEUS ( 0.927 versus 0.625 ; $\mathrm{P}=0.001)$. The accuracy and specificity of DCEUS and MDCT for Borrmann $\otimes$ were similar, but the sensitivity of MDCT was significantly higher than that of DCEUS ( $88 \%$ versus $25 \%$; Fig. 5 ). 
Table 1

The Accuracy, Sensitivity, Specificity, and Youden's Index of DCEUS and MDCT for Assessing Gross Classification of Gastric Cancer.

\begin{tabular}{|c|c|c|c|c|}
\hline & $\begin{array}{l}\text { Accuracy, } \\
\%(n)\end{array}$ & $\begin{array}{l}\text { Sensitivity, } \\
\%(n)\end{array}$ & $\begin{array}{l}\text { Specificity, } \\
\%(n)\end{array}$ & Youden's index \\
\hline \multicolumn{5}{|l|}{ DCEUS } \\
\hline EGC & $96 \%(52 / 54)$ & $75 \%(6 / 8)$ & $100 \%(46 / 46)$ & 0.75 \\
\hline Borrmann $\otimes$ & $96 \%(52 / 54)$ & $100 \%(2 / 2)$ & $96 \%(50 / 52)$ & 0.96 \\
\hline 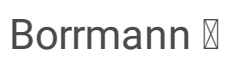 & $87 \%(47 / 54)$ & $17 \%(1 / 6)$ & $96 \%(46 / 48)$ & 0.13 \\
\hline 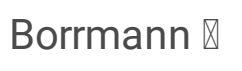 & $72 \%(39 / 54)$ & $90 \%(27 / 30)$ & $50 \%(12 / 24)$ & 0.4 \\
\hline 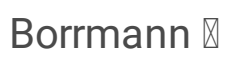 & $89 \%(48 / 54)$ & $25 \%(2 / 8)$ & $100 \%(46 / 46)$ & 0.25 \\
\hline \multicolumn{5}{|l|}{ MDCT } \\
\hline EGC & $92 \%(50 / 54)$ & $62 \%(5 / 8)$ & $98 \%(45 / 46)$ & 0.6 \\
\hline Borrmann $\otimes$ & $94 \%(51 / 54)$ & $50 \%(1 / 2)$ & $96 \%(50 / 52)$ & 0.46 \\
\hline 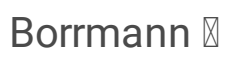 & $80 \%(43 / 54)$ & $33 \%(2 / 6)$ & $85 \%(41 / 48)$ & 0.18 \\
\hline 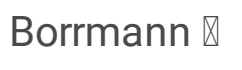 & $74 \%(40 / 54)$ & $73 \%(22 / 30)$ & $75 \%(18 / 24)$ & 0.48 \\
\hline Borrmann $\otimes$ & $96 \%(52 / 54)$ & $88 \%(7 / 8)$ & $98 \%(45 / 46)$ & 0.86 \\
\hline
\end{tabular}

Table 2

The Areas Under the ROC Curves for Each Gross Classification of Gastric Cancer by DCEUS and MDCT.

\begin{tabular}{|c|c|c|c|}
\hline \multirow[t]{2}{*}{ Pathology } & \multicolumn{2}{|l|}{ AUC(95\%Cl) } & \multirow[t]{2}{*}{$\mathrm{P}$ Value } \\
\hline & DCEUS & MDCT & \\
\hline EGC & $0.875(0.757,0.949)$ & $0.802(0.671,0.898)$ & 0.248 \\
\hline 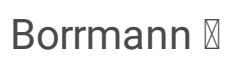 & $0.981(0.900,0.999)$ & $0.731(0.593,0.842)$ & 0.317 \\
\hline Borrmann $\otimes$ & $0.562(0.421,0.697)$ & $0.594(0.451,0.725)$ & 0.717 \\
\hline Borrmann $\otimes$ & $0.7(0.560,0.817)$ & $0.742(0.604,0.851)$ & 0.464 \\
\hline Borrmann $\otimes$ & $0.625(0.483,0.753)$ & $0.927(0.822,0.980)$ & 0.001 \\
\hline
\end{tabular}




\section{Discussion}

The precise preoperative gross classification is very critical in determining the appropriate treatment for GC. Endoscopic resection was recommended as the standard strategy for EGC without submucosal infiltration [12]. While GC of Borrmann III and IV more frequently invaded the serosal surface, involved lymph nodes and caused peritoneal metastasis than those of Borrmann I and II. Thus, the prognoses of GC of Borrmann III and IV lesions were prominently worse than those of patients with Borrmann I and II lesions [5]. Therefore, research showed gastrectomy with extended lymph node dissection and radical gastrectomy with sufficient proximal and distal distances to the primary tumor to get histological tumorfree margins were more important for patients with Borrmann III and IV lesions [5].

In this study, both DCEUS and MDCT for EGC and Borrmann I had a moderate or good evaluation capacity. The sensitivities of DCEUS for EGC and Borrmann I were higher than those of MDCT (75\% versus $62 \% ; 100 \%$ versus $50 \%$ ), although the diagnostic performance did not achieve statistical significance between them $(P=0.248 ; P=0.317)$. One case was corrected from Borrmann II on MDCT to EGC on DCEUS and one case was corrected from EGC on MDCT to Borrmann $₫$ on DCEUS. This is probably because DCEUS has higher spatial resolution for showing the gastric wall than MDCT and it could show five alternating hyper and hypoechoic layers from inside to outside as the superficial mucosa, muscularis mucosa, submucosa, muscularis propria, and serosa [13-14]. Thus DCEUS may be an optimal method in determining EGC. There were only 2 cases of the pathological Borrmann I classification, so the result may be influenced by sampling bias. In order to get more accurate results, we should increase the sample size in future studies.

In this study, both DCEUS and MDCT for Borrmann $\triangle$ classification of GC had a limited evaluation

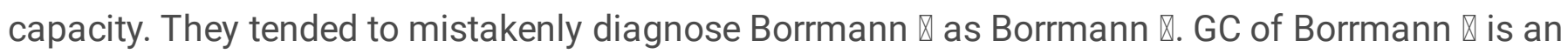
ulcerative lesion with distinct border, while Borrmann III is an ulcerating tumor with an infiltrating base. The differentiation of peritumoral inflammation and fibrosis from tumor infiltration on DCEUS and MDCT is very difficult, thus Borrmann $\otimes$ could be misdiagnosed as Borrmann $\otimes$.

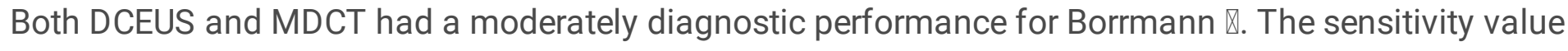
of DCEUS for Borrmann $\triangle$ was higher than that of MDCT, while the specificity value for DCEUS was lower than that for MDCT ( $90 \%$ versus $73 \% ; 50 \%$ versus $75 \%$ ). MDCT tended to mistakenly diagnose Borrmann $\triangle$ as Borrmann $\nabla$. Five cases were corrected from Borrmann $\nabla$ on MDCT to Borrmann $\triangle$ on DCEUS. Underclassification may be because the minimal infiltration of cancer cells into the gastric wall was beyond the resolution of MDCT. As mentioned above, the prognosis of Borrmann III was prominently worse than that of Borrmann II lesions and their surgical procedures might be different, so it's important to identify Borrmann III lesions sensitively rather than underestimate them. Thus, DCEUS may be a preferred method in diagnosing Borrmann III.

In this study, the ability of MDCT to diagnose Borrmann IV was significantly superior to that of DCEUS. The accuracy and sensitivity of DCEUS in the detection of Borrmann IV were obviously lower than those of MDCT ( $89 \%$ versus $96 \% ; 25 \%$ versus $88 \%$ ). The specificity values for DCEUS and MDCT were similar 
(100\% versus $98 \%$ ). Five cases were corrected from Borrmann $\triangle$ on DCEUS to Borrmann IV on MDCT. The reasons are as follows: (1) GC of Borrmann IV usually presents as a diffuse thickening of the gastric wall without a well-defined mass or ulceration. However, when the gastric wall thickens markedly, it may be mistaken for a mass. So it possibly results in being misdiagnosed as Borrmann $\mathbb{~}$. In this study, six patients with Borrmann IV were understaged as Borrmann $\triangle$ by DCEUS (6/8). (2) DCEUS can be affected by the air in the gastrointestine, shadow of ribs and xiphoid process, and artifact in the near field, so it sometimes cannot display the gastric wall, cavity and perigastric tissue well. Therefore, MDCT may be a preferred method in diagnosing Borrmann IV.

In this study, the accuracies of DCEUS and MDCT for EGC and Borrmann $₫$ were higher than those

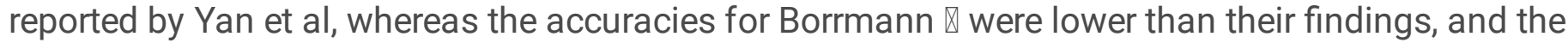
accuracies for Borrmann $\otimes$ and IV were similar to theirs [2]. Their findings indicated that the ability of DCEUS for EGC, Borrmann $₫$ and Borrmann $₫$ was superior to that of MDCT, whereas the ability of DCEUS and MDCT for Borrmann $\otimes$ and Borrmann IV were similar. However, they did not evaluate and compare the sensitivity and specificity of DCEUS and MDCT in the gross classification of GC. Thus, their data were not comparable to the data in this study.

There were some limitations to this study. First, this study was retrospective and only included patients referred to our hospital for surgery. GC histologically proved with biopsy was known before DCEUS and MDCT examinations. Second, although we collected data over 3 years, the number of patients who were

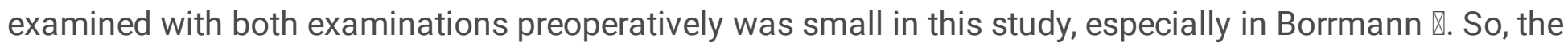
result was influenced by a sampling bias. Multicenter studies are necessary to make the results more reliable in future.

In conclusion, DCEUS may be considered as a useful complementary imaging modality to MDCT for the evaluation of the gross classification of GC preoperatively.

\section{Abbreviations}

GC Gastric cancer

EGC Early gastric cancer

AGC Advanced gastric cancer

MDCT Multidetector computed tomography

CEUS Contrast-enhanced ultrasound

AUC Area under the curve

\section{Declarations}




\section{Ethics approval and consent to participate}

All procedures performed in studies involving human participants were in accordance with the ethical standards of the institutional and/or national research committee and with the 1964 Helsinki declaration and its later amendments or comparable ethical standards. The study was approved by our institutional review board, with written informed consent obtained from each patient.

\section{Consent for publication}

Not applicable.

\section{Availability of data and materials}

The datasets used and analysed during the current study are available from the corresponding author on reasonable request.

\section{Competing interests}

The authors declared no potential conflicts of interest.

\section{Funding}

This work was financially supported by the National Key Research and Development Program of China (grant 2016 YFA0201400) and the Beijing Natural Science Foundation (grant 7182180).

\section{Authors' contributions}

HYG conceived the topic of this research and involved in acquisition of data. PH and LZ analyzed data and prepared the manuscript. LYM, TLW, JXY and LMM involved in acquisition of data. HX and FZ involved in drafting of the manuscript. BZ took part in critical revision of the manuscript. All authors read and approved the final manuscript.

\section{Acknowledgements}

Not applicable.

\section{References}

1. Hwang SW, Lee DH. Is endoscopic ultrasonography still the modality of choice in preoperative staging of gastric cancer? World J Gastroenterol 2014;20:13775-13782. doið 10.3748/wjg.v20.i38.13775.

2. Yan CX, Bao XF, Shentu WH, Chen J, Liu CM, Ye Q, et al. Preoperative Gross Classification of Gastric Adenocarcinoma: Comparison of DContrast-Enhanced Ultrasound and Multi-Detector Row CT. Ultrasound Med Biol 2016;42:1431-1440. 
3. Pan M, Huang P, Li S, et al. Dcontrast-enhanced ultrasonography in preoperative Borrmann classification of advanced gastric carcinoma: comparison with histopathology. Sci Rep 2013;3:3338.

4. Japanese Gastric Cancer A. Japanese classification of gastric carcinoma: 3rd English edition. Gastric Cancer 2011;14:101-112.

5. Li C, Oh SJ, Kim S, et al. Macroscopic Borrmann type as a simple prognostic indicator in patients with advanced gastric cancer. Oncology 2009;77:197-204.

6. Li S, Huang P, Wang Z, et al. Preoperative T staging of advanced gastric cancer using Dcontrastenhanced ultrasound. Ultraschall Med 2012;33:E218-E224.

7. Zheng Z, Yu Y, Lu M, et al. Dcontrast-enhanced ultrasonography for the preoperative evaluation of gastric cancer: a comparison to endoscopic ultrasonography with respect to histopathology. Am J Surg 2011;202:605-611.

8. Huang P, Li S, Aronow WS, et al. Dcontrast-enhanced ultrasonography evaluation of preoperative Lauren classification of advanced gastric carcinoma. Arch Med Sci 2011;7:287-293.

9. Piscaglia F, Nolsoe C, Dietrich CF, et al. The EFSUMB Guidelines and Recommendations on the Clinical Practice of Contrast Enhanced Ultrasound (CEUS): update 2011 on non-hepatic applications. Ultraschall Med 2012;33:33-59.

10. Park SH, Kim TK, Lee KH, et al. Quantitative comparison of tumor vascularity of hepatocellular carcinoma after intravenous contrast agent: conventional versus harmonic power Doppler US. Abdom Imaging 2001;26:178-183.

11. Borrmann R. Geschwulste des margens. In: Henke F, Lubarsch $O$ (eds). Handbuch spez pathol anat und hist. Berlin: Springer-Verlag; 1926. 864-871.

12. Japanese Gastric Cancer A. es 2010 (Japanese gastric cancer treatment guidelinver. 3). Gastric Cancer 2011;14:113-123.

13. Chen CY, Hsu JS, Wu DC, et al. Gastric cancer: preoperative local staging with 3D multi-detector CT-correlation with surgical and histopathologic results. Radiology 2007;242:472-482.

14. Kim HJ, Kim AY, Oh ST, et al. Gastric cancer staging at multi-detector row CT gastrography: comparison of transverse and volumetric CT scanning. Radiology 2005;236:879-885.

\section{Figures}




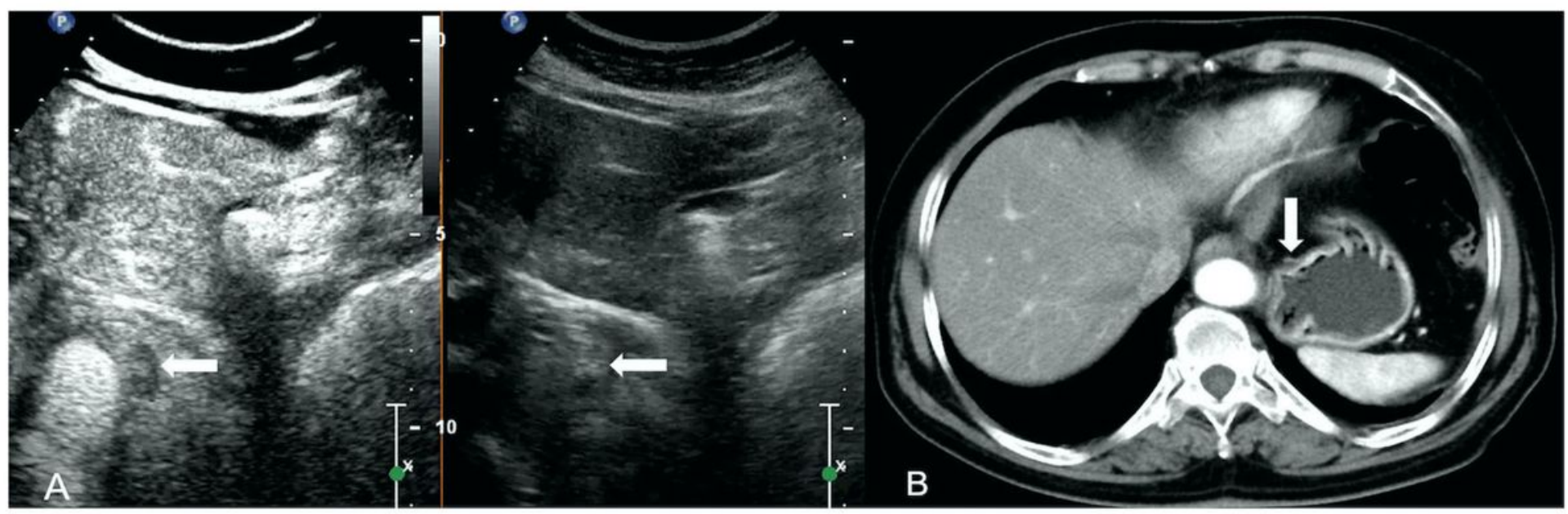

\section{Figure 1}

Gastric cancer classified as EGC in a 67-year-old woman by pathologic analysis. A The thickening of the cardia wall, limited to the submucosa (white arrows) can be seen on DCEUS, and it is classified as EGC. B Multidetector CT shows that thickening with infiltration into muscularis propria with sharply demarcated

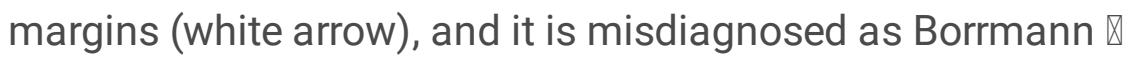
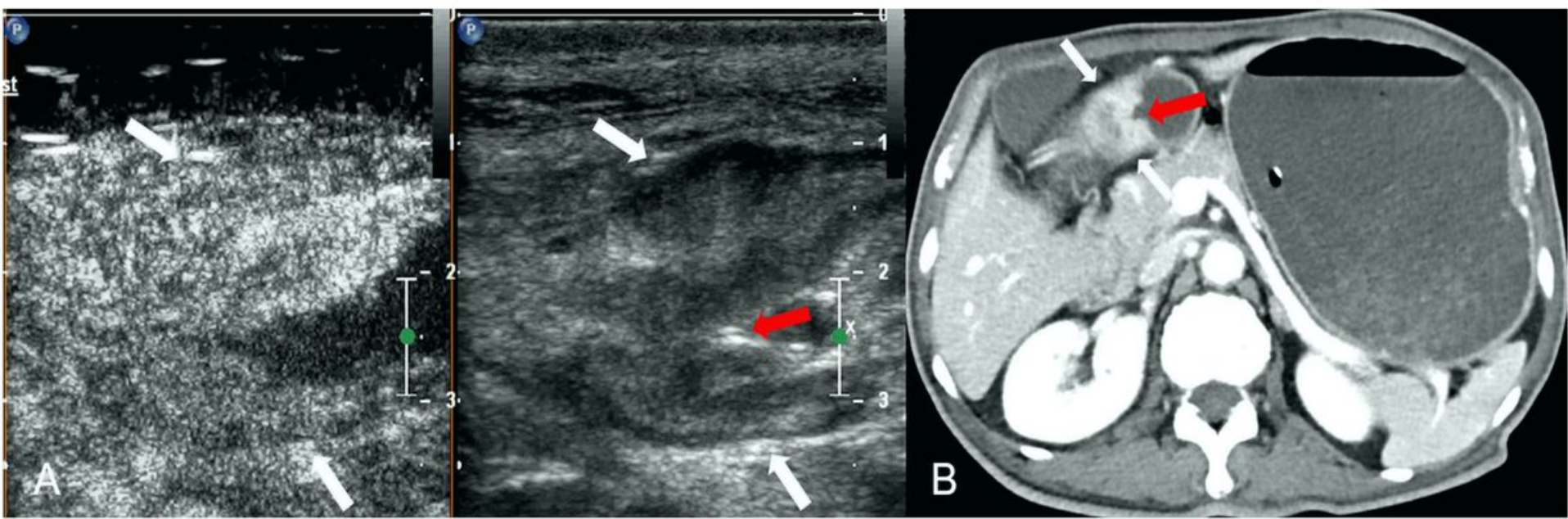

Figure 2

Gastric cancer classified as Borrmann $\nabla$ in a 41-year-old woman by pathologic analysis. A The thickened gastric wall with polypoid appearance (white arrows) can be seen on DCEUS, and it is classified as Borrmann $\nabla$. B Multidetector CT shows that thickening without infiltration into muscularis propria (white arrow), and it is misdiagnosed as EGC 


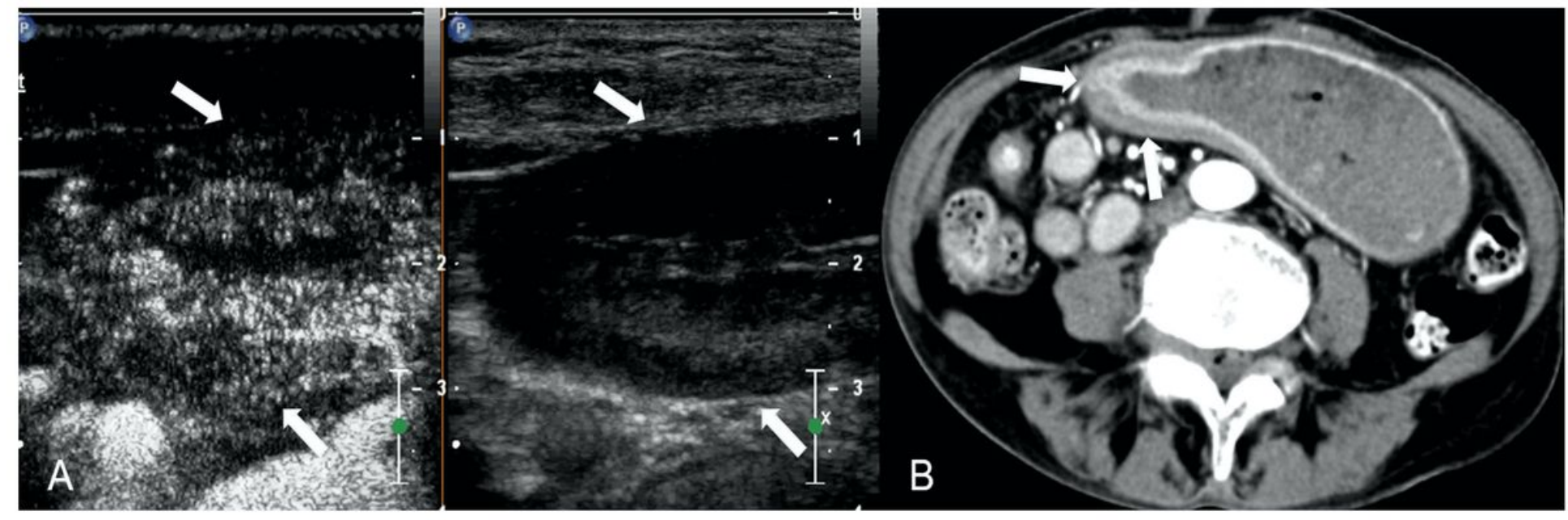

Figure 3

Gastric cancer classified as Borrmann $\otimes$ in a 70-year-old woman by pathologic analysis. A An ulcerating lesion (red arrow) without definite limits, infiltrating into the surrounding gastric wall (white arrow) can be seen on DCEUS, and it is misdiagnosed as Borrmann $\triangle$. B Multidetector CT shows an ulcerative lesion (red

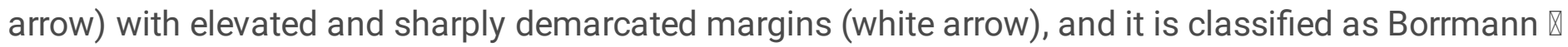
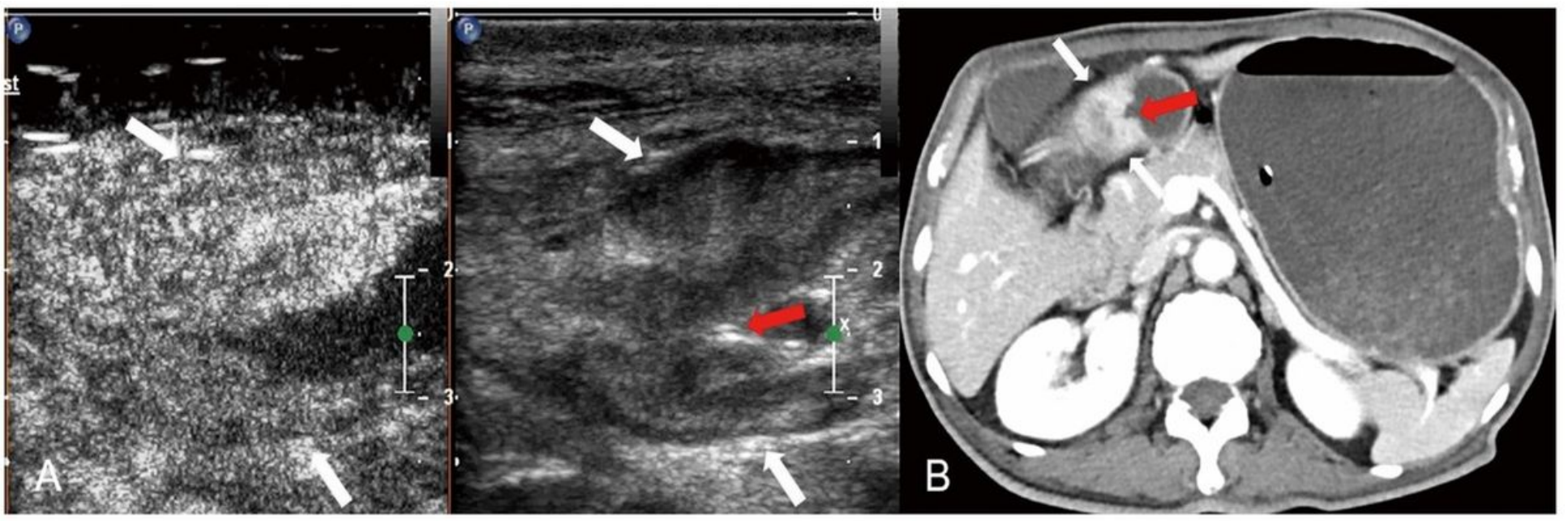

Figure 4

Gastric cancer classified as Borrmann $\triangle$ in a 66 -year-old man by pathologic analysis. A An ulcerating lesion (red arrow) without definite limits, infiltrating into the surrounding gastric wall (white arrow) can be seen on DCEUS, and it is classified as Borrmann $\triangle$. B Multidetector CT shows that an ulcerative lesion (red arrow) with elevated and sharply demarcated margins (white arrow), and it is misdiagnosed as Borrmann 


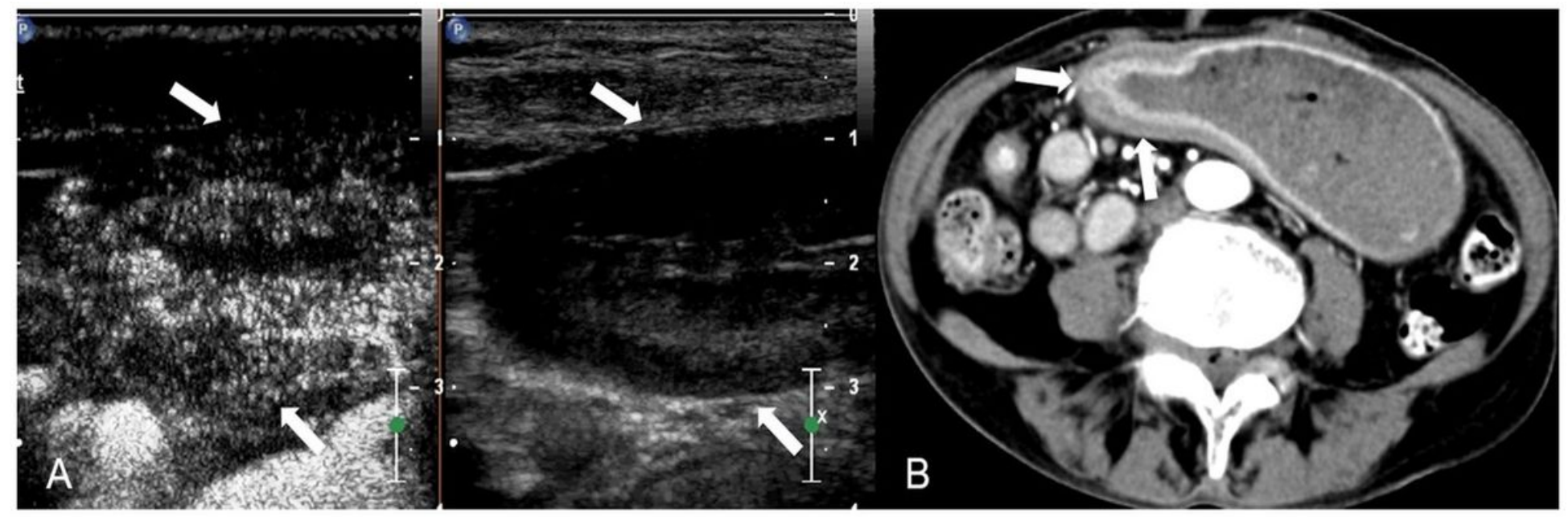

\section{Figure 5}

Gastric cancer classified as Borrmann IV in a 67-year-old woman by pathologic analysis. A An ulcerating lesion without definite limits, infiltrating into the surrounding gastric wall (white arrow) can be seen on

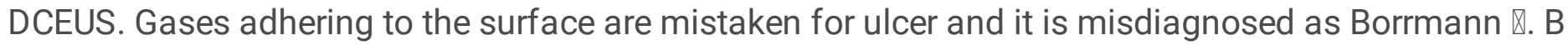
Multidetector CT shows that diffusely infiltrating tumor without ulcer or a discretely marginated mass (white arrow), and it is classified as Borrmann IV 